\title{
Ion Beam Sputtering for Controlled Synthesis of Thin MAX (MXene) Phases
}

Pavel Horak $^{1}$, Jiri Vacik ${ }^{1 *}$, Snejana Bakardjieva ${ }^{2}$, Antonino Cannavo ${ }^{1}$, Giovanni Ceccio ${ }^{1}$, Jaroslav Kupcik $^{2}$, Robert Klie ${ }^{3}$

1. Nuclear Physics Institute (NPI) of the Czech Academy of Sciences (CAS), 205 68, Czech Republic.

2. Institute of Inorganic Chemistry of the Czech Academy of Sciences, 20568 Rez, Czech Republic.

3. University of Illinois at Chicago, Department of Physics, 845 W Taylor Str., 60607 Chicago, USA.

* Corresponding author: vacik@ujf.cas.cz

There is growing interest in synthesis of thin functional films with tailored properties. This requires adequate deposition techniques, as well as a proper control diagnostic. Ion Beam Sputtering (IBS), as a physical vapor deposition (PVD) method, is capable to fulfill technological challenges. It is mainly because in IBS (in contrast to other PVD techniques) the production of ions in an ion source, the generation of sputtered particles at the targets, and the growth of thin films on substrates are spatially separated. Thus, by changing of ion beam characteristics (i.e., ion species, energy, and intensity) and/or parameters of a target system (ion incident or emission angles), the deposition conditions for thin film growth can be finely tuned and modified.

In the CANAM research infrastructure of the NPI in Rez [1], a new system (LEIF - Low Energy Ion Facility) has been recently assembled (Fig. 1) utilizing a new-type of a multi-CUSP ion source. It can produce ions in a broad, tunable energy range $100 \mathrm{eV}-35 \mathrm{keV}$ with a high current up to $500 \mu \mathrm{A}$. This system was adapted to employ an IBS technique, and it is also used for ion irradiation/implantation (in an implantation chamber) with high fluences up to $10^{20} \mathrm{~cm}^{-2}$. In the process of ion beam sputtering, the targets are mounted on a cooled $\mathrm{Cu}$ holder (see Fig. 1, detail of the sputtering chamber). In the case of a multiphase composite synthesis, the target holder acquires a multi-angle form that is revolving according the required stoichiometric ratio and sputtering/deposition rates of the phases. For promotion of the phase synthesis, the substrates are fixed on a heated platform and kept at elevated temperatures.

Using the LEIF system, various thin films of MAX (and MXene) phases have been prepared, and by ion bombardment radiation tolerance of the films has been tested. The MAX phases are a new class of ternary carbides or nitrides with a stoichiometric nomenclature $\mathrm{M}_{\mathrm{n}+1} \mathrm{AX}_{\mathrm{n}},(n=1,2,3)$, where $\mathrm{M}$ is a transition metal, $\mathrm{A}$ is an element from the IIIA or IVA groups of the periodic chart, and $\mathrm{X}$ is carbon or nitrogen [2]. MXenes consist of a few atoms-thick layers of transition metal carbides or carbonitrides [3]. Both phases are potentially highly promising materials because of remarkable combination of their chemical, physical, electrical, and mechanical properties. They are favorable candidates for applications in extreme conditions, e.g., in the next generation of fission and fusion reactors [4].

Figure 2 shows an example of a series of the HRTEM micrographs (measured at the UIC Chicago) of a $\mathrm{Ti}_{2} \mathrm{SnC}$ phase. The $60 \mathrm{~nm}$ thin films were prepared by the $25 \mathrm{keV} / 400 \mu \mathrm{A} \mathrm{Ar}{ }^{+}$ion beam on Ni mesh substrates kept during the deposition at $150{ }^{\circ} \mathrm{C} / 5$ hours. The post-deposition bombardment was performed by the $30 \mathrm{keV} / 100 \mathrm{nA} \mathrm{Ar}{ }^{+}$ion beam up to the fluence $10^{15} \mathrm{~cm}^{-2}$. The HRTEM images shows synthesis of separated, individual crystallites of the $\mathrm{Ti}_{2} \mathrm{SnC}$ phase (a) emerged in the amorphous surroundings (b). The forceful irradiation of the films led to aggregation of the $\mathrm{Ti}_{2} \mathrm{SnC}$ crystallites (c) and partial amorphization of their surface layers (d). The results show that by IBS the MAX (MXene) 
phases can be successfully synthesized, and that the inspected MAX phases show suitability for harsh radioactive environment.

\section{References:}

[1] http://canam.ujf.cas.cz (accessed 02, 22, 2019).

[2] P Eklund, M Beckers, U Jansson, H Högberg, L Hultman, Thin Solid Films 518 (2010), p. 1851.

[3] M Naguib, V Mochalin, M Barsoum, Y Gogotsi, Advanced Materials 26 (2011), p. 992.

[4] D Tallman, "On the Potential of MAX phases for Nuclear Applications“, Thesis, Drexel Univ., 2015.

[5] The authors acknowledge funding from the MEYS, Czech Republic, Project LTAUSA 17128.

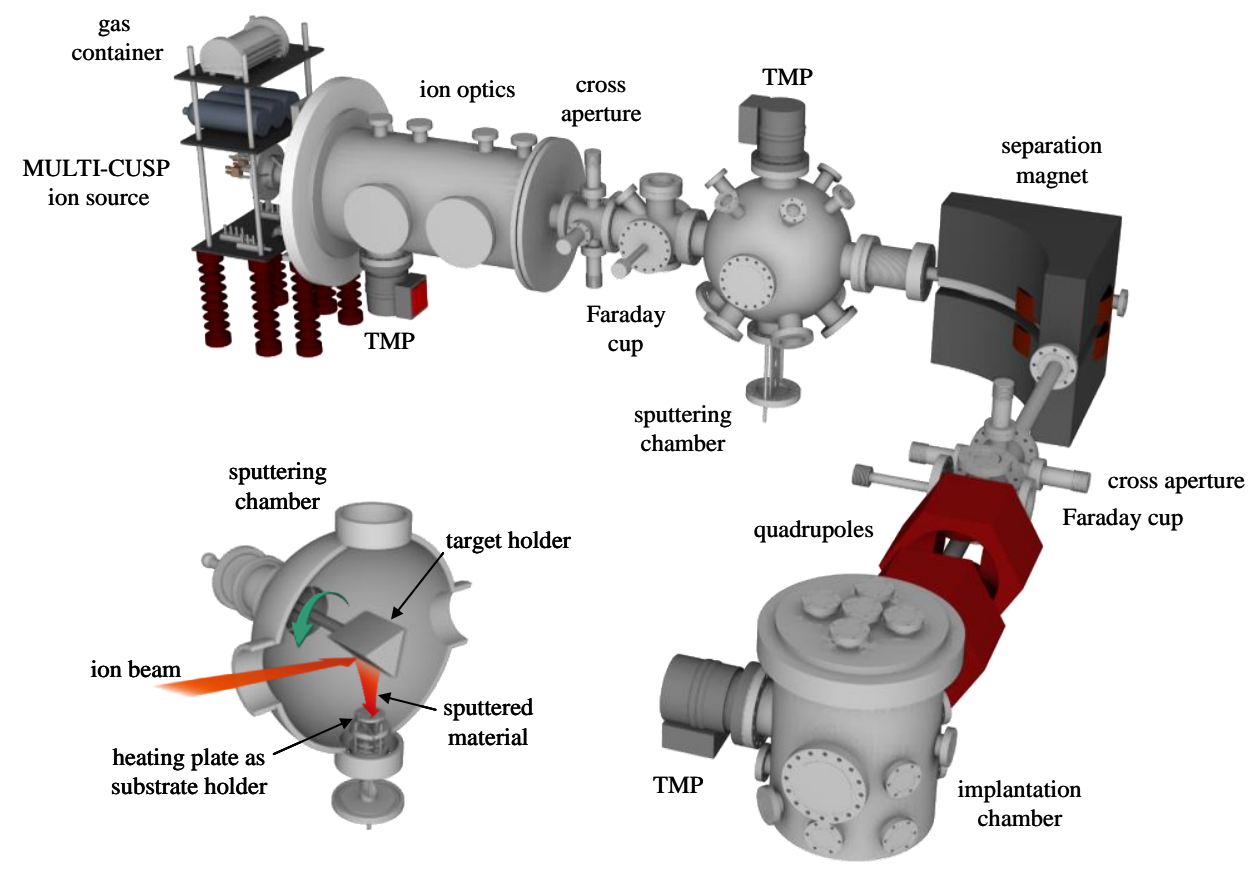

Figure 1. Low Energy Ion Facility (LEIF) assembled in the NPI CANAM infrastructure for fabrication of thin films (of MAX and MXene phases) and their irradiation by an intense ion beam.

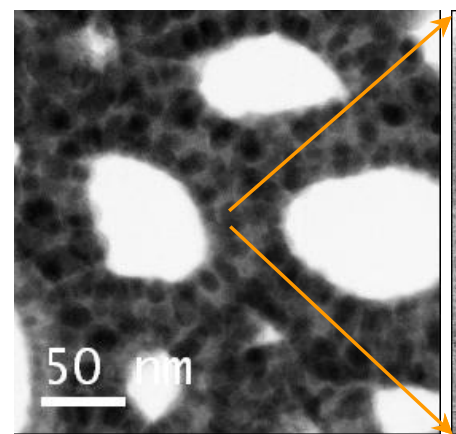

(a)

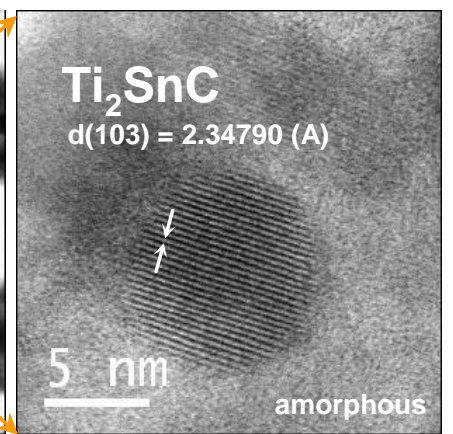

(b)

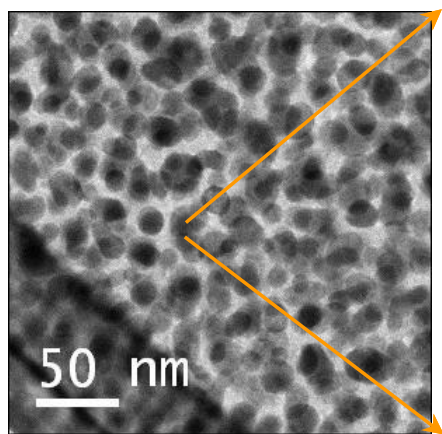

(c)

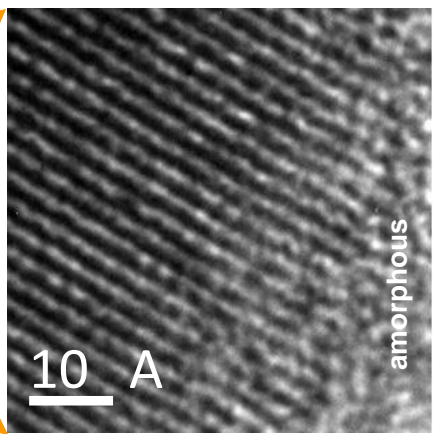

(d)

Figure 2. Synthesis of a thin $\mathrm{Ti}_{2} \operatorname{SnC}$ film (a, b) by IBS $\left(25 \mathrm{keV} \mathrm{Ar}^{+} / 400 \mu \mathrm{A}\right)$, and its micro-structural modification (c, d) by ion beam irradiation $\left(30 \mathrm{keV} \mathrm{Ar}^{+} / 100 \mathrm{nA}, 10^{15} \mathrm{~cm}^{-2}\right)$. 